\title{
COMPUTER SIMULATIONS OF IRON IN MAGNESIUM SILICATE PEROVSKITE
}

\author{
Kate Wright and Geoffrey D. Price
}

Department of Geophysical Sciences, University College London.

\begin{abstract}
We use atomistic computer simulation techniques to investigate the site partitioning of iron in $(\mathrm{Mg}, \mathrm{Fe}) \mathrm{SiO}_{3}$ perovskites. Our calculations predict that the most energetically favourable reaction for iron substitution will be a direct exchange of $\mathrm{Fe}^{2+}$ for $\mathrm{Mg}^{2+}$. Substitution of $\mathrm{Fe}$ into the octahedral site and $\mathrm{Si}$ into the 8-12 fold coordinated site, as proposed by Jackson et al. [1987], is predicted to be extremely unlikely.
\end{abstract}

\section{Introduction}

Magnesium silicate perovskite is considered to be the most abundant phase in the Earth's lower mantle. Over the last decade, significant advances in technology have led to the production of single crystals of $\mathrm{MgSiO}_{3}$ perovskite, which has allowed the structure of this important phase to be refined [Horiuchi et al., 1987], and its thermal expansion and elastic moduli to be measured [Knittle et al., 1986; Ross and Hazen, 1989; Yeganeh-Haeri et al., 1989]. Nevertheless, many of the physical and thermochemical properties of this phase still remain unknown.

The probable composition of mantle perovskites, and the location of substitutional elements within the perovskite structure has attracted considerable attention. The location of iron (which could account for as much as $5-10 \%$ by weight of the lower mantle) within the perovskite structure, has been of particular interest. Experiments have shown that the iron in the lower mantle will preferentially partition into magnesiowustite [e.g. Ito and Yamada, 1982], and that for $x>0.25,\left(\mathrm{Mg}_{1-\mathrm{x}} \mathrm{Fe}_{\mathrm{x}}\right) \mathrm{SiO}_{3}$ perovskite appears to be unstable with respect to simple oxides.

Jackson et al. [1987] have carried out extended X-ray absorption fine structure (EXAFS) and X-ray absorption near-edge (XANES) studies on $\mathrm{Fe}$ bearing $\mathrm{MgSiO}_{3}$ perovskite. Their results led them to conclude that iron in magnesium silicate perovskite would preferentially partition into the octahedral site normally occupied by silicon, rather than replace $\mathrm{Mg}$ on the larger, 8-12 fold coordinated site in the perovskite structure. They further suggested, that the displaced silicon would occupy the 8-12 fold coordinated site normally occupied by divalent ions. The details of this crystal chemistry have recently been elaborated upon by Williams et al. [1989].

The above proposed cation distribution represents an unusual crystal chemical situation, with a reversal of the cation site occupancy that might be intuitively expected. In this study, therefore, we have carried out a series of computer simulations to test the above scenario. We have used an atomistic approach based on the Born model of solids, where a potential function represents the interactions between atoms or ions in the system, to investigate the energy of a variety of cation substitution reactions within a predominantly pure $\mathrm{MgSiO}_{3}$ perovskite.

In the following sections the methodology used in this study of the location of $\mathrm{Fe}$ in the perovskite lattice will be briefly described, and the basis of the defect energy calculations

Copyright 1989 by the American Geophysical Union.

Paper number 89GL03362.

0094-8276/89/89GL-03362\$03.00 outlined. Our findings are then compared with experimental results and their significance discussed.

\section{Computer Simulation Techniques}

The nature of the cation distribution in a crystal and the role of minor substitutional elements, can be investigated by performing calculations on a variety of cation configurations or local "defect structures". The exchange of cations between distinct crystallographic sites that result in a predicted destabilization of the lattice will be unlikely to occur. The energy of such defect reactions can be obtained, in principle, from accurate atomic scale modelling. Modelling techniques used in cation distribution or defect studies can range from quantum mechanical calculations, involving the direct solution to Schrodinger's equation, to atomistic simulation techniques. In these latter methods, an algorithm or interatomic potential is defined to describe the total energy of the system in terms of atomic positions. The lattice energy includes contributions from ionic, covalent and van der Waals type bonding. Such models have been widely and successfully used to model both the perfect lattice, thermodynamic properties of materials [e.g. Price et al., 1987a, b], as well as quantitatively predicting their point defect and diffusional properties [e.g. Catlow, 1977].

For ionic or semi-ionic solids, the dominant component of the cohesive energy, $U_{T}$, will be the electrostatic or Coulombic term, $\mathrm{U}_{\mathrm{C}}$. However, as ions are not simple point charges, it is necessary to take into account the short range repulsive interactions, $U_{i j}$, produced by the overlap of nearest neighbour electron clouds. This short-range component is well modeled by the Buckingham potential:

$$
U_{i j}=\sum_{i j} A_{i j} \exp \left(-r_{i j} / B_{i j}\right)-C_{i j} r_{i j}^{-6}
$$

where $A, B$ and $C$ are parameters obtained either from fitting to ab initio energy surfaces, or empirically by fitting to crystalline properties.

In this study we have used the potential THB1, which has previously been used successfully to model the perfect lattice and defect properties of forsterite [Price et al., 1987a, b; Price et al., 1989] and the defect properties of pure $\mathrm{MgSiO}_{3}$ perovskite [Wall and Price, 1989]. THB1 is composed of terms originally developed to model simple binary oxides. The O-O short range parameters were derived theoretically using Hartree-Fock methods [Catlow, 1977], while those for $\mathrm{Mg}-\mathrm{O}$ and $\mathrm{Si}-\mathrm{O}$ [Sanders et al., 1984] were fitted to experimental data. When modelling defects, it is essential to describe the local polarization effects cause by the defect, and so in our models oxygen polariziblity is described via a simple harmonic shell model. Additionally, we have used potentials for $\mathrm{Fe}^{2+}-\mathrm{O}$ and $\mathrm{Fe}^{3+}-\mathrm{O}$ interactions developed by Lewis and Catlow [1985]. The full set of potential parameters used in this study are listed in Table 1 . Our potentials assume full ionic charges. We believe that the use of such a fully ionic model to describe $\mathrm{MgSiO}_{3}$ perovskite is well justified, since ab initio calculations by Cohen et al. [1989] showed little evidence for covalent bonding in this phase.

In our study of defect energies and cation distribution in perovskite, we have used the computer code CASCADE, 
TABLE 1. Potential parameters used to calculate defect energies. (Short range cut off $=11.95 \AA$ )

$\begin{array}{ll}\mathrm{q}_{\mathrm{Mg}} & +2.0 \\ \mathrm{q}_{\mathrm{Fe}(2+)} & +2.0 \\ \mathrm{q}_{\mathrm{Fe}(3+)} & +3.0 \\ \mathrm{q}_{\mathrm{Si}} & +4.0 \\ \mathrm{q}_{\mathrm{O}(\text { shell) }} & -2.848 \\ \mathrm{q}_{\mathrm{O}(\text { core })} & +0.848 \\ \mathrm{~A}(\mathrm{eV}) & \\ \mathrm{Mg}-\mathrm{O} & 1428.5 \\ \mathrm{Fe}^{2+}-\mathrm{O} & 1207.6 \\ \mathrm{Fe}^{3+}-\mathrm{O} & 1102.4 \\ \mathrm{Si}-\mathrm{O} & 1283.9 \\ \mathrm{O}-\mathrm{O} & 22764.3 \\ & \\ \mathrm{~B}(\AA) & \\ \mathrm{Mg}-\mathrm{O} & 0.2945 \\ \mathrm{Fe}-\mathrm{O} & 0.3084 \\ \mathrm{Fe} & -\mathrm{O} \\ \mathrm{Si}-\mathrm{O} & 0.3299 \\ \mathrm{O}-\mathrm{O} & 0.3205 \\ & 0.1490 \\ \mathrm{C}\left(\mathrm{eVA} \AA^{6}\right) & \\ \mathrm{O}-\mathrm{O} & \\ \mathrm{Si}-\mathrm{O} & 27.88 \\ \mathrm{k}^{\mathrm{B}} \mathrm{eVA} \AA^{-2} & 10.66 \\ \mathrm{k}^{\mathrm{s}} \mathrm{eVrad} & \\ \end{array}$

supported by the British Science and Engineering Research Council, to evaluate the energy of the configurations of interest. In ionic materials, defects are charged species and cause long range disruption in the lattice. These long-range perturbations can be effectively modelled by the Mott and Littleton [1938] approach, in which the crystal is divided into two spherical regions (Fig. 1). In region 1, which contains the defect at its centre, an explicit atomistic simulation is carried out to adjust the coordinates of all ions within the region until they are at positions at which no net forces act on them, i.e. they are relaxed around the defect. In region 2, the effects of the defect are relatively weak and the relaxation can be treated by more approximate means. Using the MottLittleton approach, a defect with charge $q$ causes a polarization $\mathbf{P}$, at a point $\mathbf{r}$ according to:

$$
P=q r^{-3}\left(1-\epsilon_{0}^{-1}\right) r
$$

where $\epsilon_{0}$ is the static dielectric constant. For the two region approximation, the total energy required for defect formation is given by:

$$
E_{D}=E_{1}(x)+E_{2}(x, y)+E_{3}(y)
$$

where $E_{1}$ is the energy of interaction of atoms in region 1 with coordinate vector $\mathrm{x}, \mathrm{E}_{3}$ the energy of ions with displacement $y$ in region 2 , and $E_{2}(x, y)$ describes the interaction between the two regions.

When $y$ is sufficiently small, as is generally assumed, $E_{3}$ can be expressed using the harmonic approximation as:

$$
E_{3}=1 / 2 \mathbf{y}^{T} \cdot \mathbf{A} \cdot \mathbf{y}
$$

where $\mathbf{A}$ is the force constant matrix. At equilibrium:

$$
\left(\mathrm{dE}_{2}(\mathrm{x}, \mathrm{y}) / \mathrm{dy}\right) \mathbf{x}=-\mathbf{A} \cdot \mathbf{y}
$$

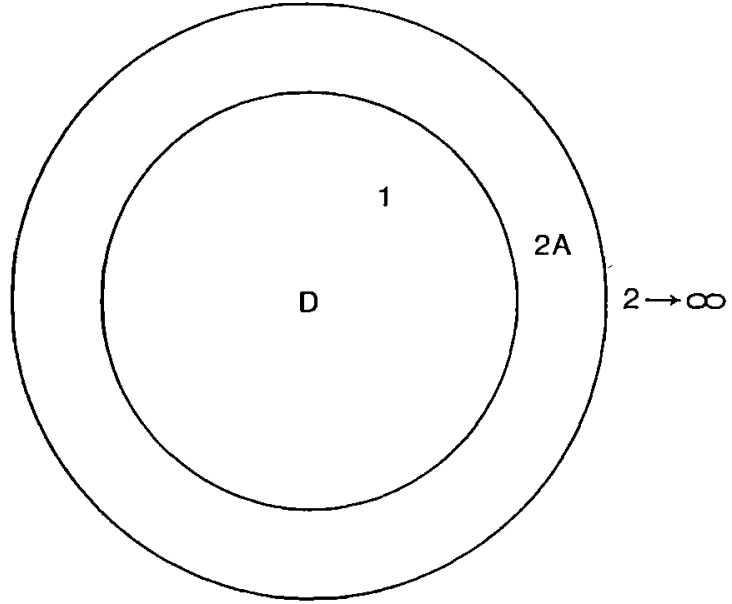

Fig 1. Schematic representation of the defect model used in this study. The defect is centred in region 1 , in which explicit atomistic relaxations are allowed.

Therefore $E_{D}$, in equation 3, can be expressed in terms of $E_{1}$, which is calculated by explicit summation, and $E_{2}$ and its derivatives. For the calculation of $\mathrm{E}_{2}$ and its derivatives, it is necessary to define a subdivision of region 2 , region $2 A$, where the interaction of ions with the defect in region 1 are considered in more detail. For the remainder of region 2 , the Mott-Littleton calculations assume that displacements are due only to the net charges on the defect. The resulting defect energy is a measure of the perturbation by the defect of the static lattice energy of the crystal. In this approach, therefore, no account is taken of zero-point energy or entropic effects. For further details of the method see Catlow [1986].

\section{Results and Discussion}

We have simulated the substitution of $\mathrm{Fe}$ into various sites in the $\mathrm{MgSiO}_{3}$ perouskite lattice, by assessing the energies of several defect reactions of the type:

$$
\mathrm{FeO}+\mathrm{Mg}_{\mathrm{Mg}}-->\mathrm{Fe}_{\mathrm{Mg}}+\mathrm{MgO}
$$

where $\mathrm{Fe}$ in the binary oxide substitutes for $\mathrm{Mg}$ on the $\mathrm{Mg}$ or 8-12 fold coordinated site in perovskite. In calculating the energetics of such substitutional reactions, we have evaluated the necessary isolated vacancy and substitutional defect energies, as well as the energies of certain bound defect pairs listed in Table 2. These values were used to calculate the energies associated with the defect reactions given in Table 3. Since we are concerned with the role of $\mathrm{Fe}$ in the perovskite lattice at high pressure, we have performed calculations at a simulated pressure of $60 \mathrm{GPa}$, as well as at $0 \mathrm{GPa}$. The high pressure simulation is based upon energy minimised output from the computer code PARAPOCS [Parker and Price 1989]. The calculations were performed on the fully relaxed, orthorhombically distorted perovskite, but the energetics of defects involving crystallographically distinct oxygens were found to be virtually identical, and so have not been differentiated. In our simulations, we used a region 1 radius of $7.2 \AA$ containing approximately 183 ions. A region 1 of this size gave a convergence in the calculated defect energy of better than $0.05 \mathrm{eV}$.

The nature of intrinsic defects in $\mathrm{MgSiO}_{3}$ perovskite have been discussed by Wall and Price [1989]. For the substitution of $\mathrm{Fe}^{2+}$ into $\mathrm{MgSiO}_{3}$ perovskite, we have considered four reactions (nos. 1-4 in Table 3). Of these, reaction 1, in which 
TABLE 2. Vacancy (V), interstitial and lattice energies (in $\mathrm{eV})$ at $0 \mathrm{~K} 0 \mathrm{GPa}$ and $0 \mathrm{~K} 60 \mathrm{GPa}$.

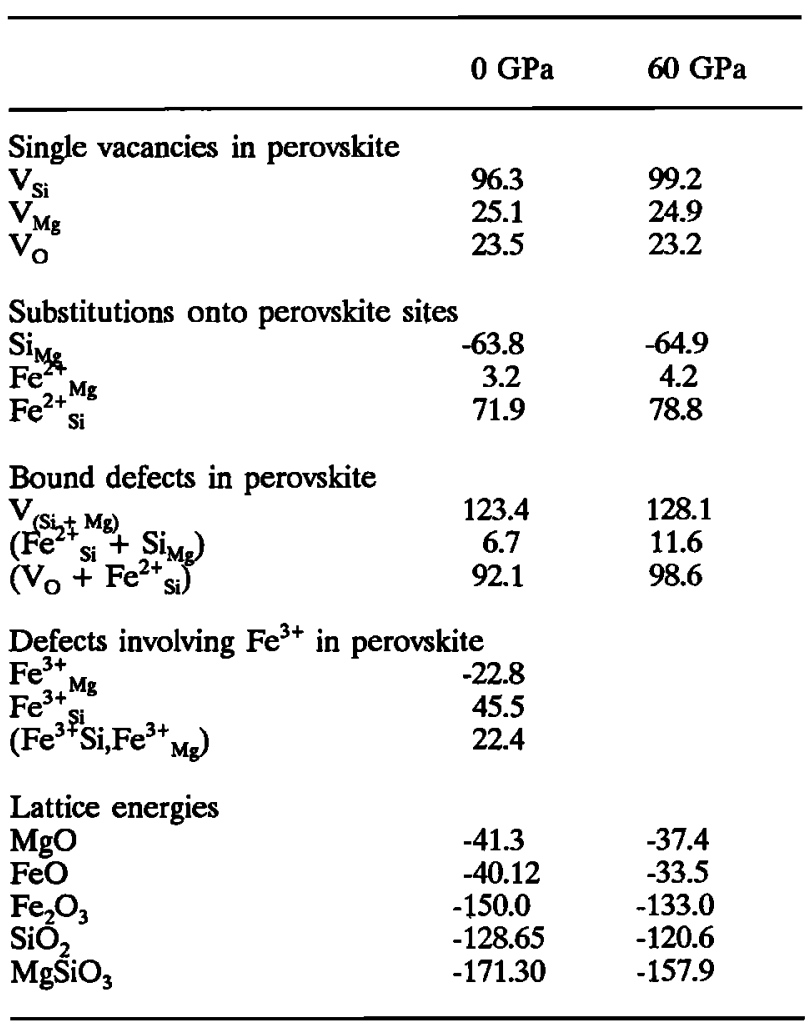

TABLE 3. Defect reactions and energies (in eV) in $\mathrm{MgSiO}_{3}$ at 0 and $60 \mathrm{GPa}$.

Reaction with FeO

$0 \mathrm{GPa} 60 \mathrm{GPa}$



Reaction with $\mathrm{Fe}_{2} \mathrm{O}_{3}$

$$
\begin{aligned}
& \text { 5) } \begin{aligned}
\mathrm{Fe}_{2} \mathrm{O}_{3}+3 \mathrm{Mg}_{\mathrm{Mg}}-\cdots> & 2 \mathrm{Fe}_{\mathrm{Mg}}+\mathrm{V}_{\mathrm{Mg}} \\
& +3 \mathrm{MgO}
\end{aligned} \\
& \text { 6) } \quad-->2 \mathrm{Fe}_{\mathrm{Si}}+\mathrm{V}_{\mathrm{o}}+2 \mathrm{SiO}_{2} \\
& \text { 7) } \quad-->\left(\mathrm{Fe}_{\mathrm{Si}}, \mathrm{Fe}_{\mathrm{Mg}}\right) \\
& +\mathrm{MgSiO}_{3} \\
& \text { 8) } \\
& \begin{aligned}
---> & 2 \mathrm{Fe}_{\mathrm{Si}}+\mathrm{Si}_{\mathrm{Mg}_{\mathrm{B}}} \\
+ & \mathrm{MgSiO}_{3}
\end{aligned}
\end{aligned}
$$

a simple exchange of $\mathrm{Mg}^{2+}$ and $\mathrm{Fe}^{2+}$ occurs, is predicted to have the lowest energy, and so on static lattice energy grounds alone would be predicted to be the most likely means of introducing $\mathrm{Fe}$ into the perovskite structure. The situation envisaged by Jackson et al. [1987] could be described by reactions 2 and 3 . The former being for isolated exchanges and the latter for bound defects. Both reactions are predicted to be extremely implausible with formation energies of 6.9 and $5.5 \mathrm{eV}$ respectively at $0 \mathrm{GPa}$. There is a small decrease in energy if the defects involved are bound, but not enough to make this a viable process. The effect of pressure on this system is to make the substitution of $\mathrm{Fe}^{2+}$ into the octahedral site even less energetically favourable. In the fourth reaction, $\mathrm{Fe}^{2+}$ substitutes for $\mathrm{Si}$ in the presence of an oxygen vacancy, and thus obviates the need for $\mathrm{Si}$ to enter the 8-12 fold coordinated site. However, although this represents a more favourable means of substituting $\mathrm{Fe}$ for $\mathrm{Si}$, the formation energy of $3.6 \mathrm{eV}$ at $0 \mathrm{GPa}$ is still much higher than for reaction 1, and again is still further destabilised by increasing pressure. From Table 2, it can be seen that the formation of a Si vacancy requires a large amount of energy and therefore any reaction involving $\mathrm{Si}$ vacancies will be extremely unfavourable.

Also included in Table 3 are reactions describing the substitution of $\mathrm{Fe}^{3+}$ into $\mathrm{MgSiO}_{3}$ at $0 \mathrm{GPa}$. These reactions (5-8 in table 3) are more complex than those involving $\mathrm{Fe}^{2+}$. Although these reactions are unlikely to occur in the more reducing conditions of the lower mantle, they are considered here because it is conceivable that $\mathrm{Fe}^{3+}$ may have been generated under experimental conditions. Reaction 7, where both $\mathrm{Si}$ and $\mathrm{Mg}$ are replaced by $\mathrm{Fe}^{3+}$ is predicted to be the most favourable with a formation energy of $1.1 \mathrm{eV}$. All the other reactions considered have energies associated with them which make them highly unfavourable. It proved impossible to simulate the role of $\mathrm{Fe}^{3+}$ in perovskite at high $\mathrm{P}$, because the highly charged cation when substituted for $\mathrm{Mg}$ in the compressed structure caused excessive disruption, and prevented the calculations from converging.

So far, we have ignored any effect that crystal field stabilization energies (CFSE) may have on the reactions considered. In the high spin state, $\mathrm{Fe}^{3+}$ enjoys no CFSE, but $\mathrm{Fe}^{2+}$ will be associated with a finite CFSE when in octahedral coordination. For simplicity we may assume that the CFSE in the more nearly spherically symmetric $8-12$ fold perovskite site is negligible. Although being dependent upon the details of the octahedral environment, the CFSE for $\mathrm{Fe}^{2+}$ in octahedral coordination is typically of the order of $0.5 \mathrm{eV}$. This is too small an energetic perturbation to affect the conclusions drawn concerning reactions $2-4$ in Table 3 . However, the role of CFSE may well be significant when assessing the reasons for the instability of $\mathrm{Fe}$ rich silicate perovskites.

\section{Conclusions}

Within the confines of the assumptions that zero-point energy and entropic terms will have a negligible effect on the energetics of defect formation, and that Fe occurs only as a di- or trivalent cationic species, we conclude:

(i) that it is energetically unfavourable, and hence improbable that $\mathrm{Fe}^{2 f}$ substitutes for $\mathrm{Si}$ in the silicate perovskite structure.

(ii) that it is energetically improbable that $\mathrm{Si}$ will substitute into the large 8-12 fold coordinated site in perovskite.

If $\mathrm{Fe}$ is to occur in the octahedral site in silicate perovskites, it will be as $\mathrm{Fe}^{3+}$. But such octahedral substitutions will always be balanced by an equal number of $\mathrm{Fe}^{3+}$ ions in the larger 8-12 fold coordinated site. Thus, our calculations suggest that the model proposed by Jackson et al. [1987] is highly unlikely, and that the most favourable reaction for the substitution of $\mathrm{Fe}$ into $\mathrm{MgSiO}_{3}$ is predicted to be Fe-Mg exchange.

It is possible to speculate that the results of the experiments carried out by Jackson et al. [1987] might be explained if the Fe in their sample had partitioned into an amorphous phase 
or in an oxide phase, rather than into the perovskite Transmission electron microscopy (TEM) of products produced in diamond anvil cell show a complex behaviour [Madon et al., 1989], in which multiphase, possibly metastable assemblages, are frequently produced. We must conclude that further more careful experimental studies are required to resolve the apparent conflict between experiment and the calculations presented here.

Acknowledgments. The authors would like to thank Richard Catlow, Stephen Parker and Alison Wall for many stimulating discussions. $\mathrm{KW}$ thanks the NERC for the receipt of a research studentship. GDP acknowledges the receipt of NERC grant GR3/6970.

\section{References}

Catlow, C.R.A., Point defect and electronic properties of uranium dioxide. Proc. $R$. Soc. London A353, 533-561, 1977.

Catlow, C.R.A., Computer simulation of defects in solids. In: Defects in solids: Modern techniques. (Eds. Chadwick, A.V. and Terenzi), pp 269-302, Plenum Press, New York, 1986.

Cohen, R., Boyer, L., Mehl, M., Pickett, W. and Krakauer, H., Electronic structure and total energy calculations for oxide perovskites and superconductors. In: Perovskite. (Eds. A. Navrotsky and D.J. Weidner). Monograph 45, pp 55-66. Amer. Geophys. Union, Washington DC, 1989.

Horiuchi, H., Ito, E. and Weidner, D.J. Perovskite-type $\mathrm{MgSiO}_{3}$ : Single-crystal X-ray diffraction study. Amer. Mineral., 72, 357-360, 1987.

Ito, E. and Yamada, H., Stability Relations of Silicate Spinels, Ilmenites and Perovskites. In: High Pressure Research in Geophysics. (Eds. S. Akimoto and M.H. Manghnani) Advances in Earth and Planet. Science, 12, pp 405-420 Reidel, Dordrecht, 1982.

Jackson, W., Knittle, E., Brown, G. and Jeanloz, R., Partitioning of $\mathrm{Fe}$ in $(\mathrm{Mg}, \mathrm{Fe}) \mathrm{SiO}_{3}$ perovskite: evidence for unusual geochemistry in the Earth's lower mantle. Geophys. Res. Letts. 14, 224-226, 1987.

Knittle, E., Jeanloz, R. and Smith, G.L., Thermal expansion of silicate perovskite and stratification of the Earth's mantle. Nature 319, 214-21, 1986.

Lewis, G.V. and Catlow, C.R.A., Potential models for ionic oxides. J. Phys. C: Solid State Phys. 18, 1149-1161, 1985.

Madon, M., Guyot, F., Peyronneau, J. and Poirier, J.P., Electron microscopy of high pressure phases synthesized from natural olivine in diamond anvil cell. Phys. Chem. Mins. 16, 320-330, 1989.

Mott, N. F. and Littleton, M.J., Conduction in polar crystals. I: Electrolyte conduction in solid salts. Trans. Farad Soc., 34, 485-499, 1938.

Parker, S.C. and Price, G.D., Computer modelling of phase transitions in minerals. (Adv.in Solid State Chem., 1, 295327, 1989.

Price, G.D., Parker, S.C. and Leslie, M., The lattice dynamics of forsterite. Min. Mag. 51, 157-170, 1987a.

Price, G.D., Parker, S.C. and Leslie, M., The lattice dynamics and thermodynamics of the $\mathrm{Mg}_{2} \mathrm{SiO}_{4}$ polymorphs. Phys. Chem. Mins. 15, 181-190, 1987b.

Price, G.D., Wall, A. and Parker, S.C., The properties and behaviour of mantle minerals: a computer simulation approach. Phil. Trans. $R$. Soc. Lond., A328, 391-407, 1989.

Ross, N.L. and Hazen, R., Single crystal X-ray diffraction study of $\mathrm{MgSiO}_{3}$ perovskite from 77-400 K. Phys. Chem. Mineral., 16, 415-420, 1989.

Sanders, M.J., Leslie, M. and Catlow, C.R.A., Interatomic potentials for $\mathrm{SiO}_{2}$ J. Chem. Soc. Comm., pp 1271-1274, 1984.

Wall, A. and Price, G.D., Defects and diffusion in $\mathrm{MgSiO}_{3}$ perovskite: A computer simulation. In: Perovskite. (Eds A. Navrotsky and D.J. Weidner). Monograpgh 45, pp 45-53. Amer. Geophys. Union, Washington DC, 1989.

Williams, Q., Knittle, E. and Jeanloz, R., Geophysical and crystal chemical significance of $(\mathrm{Mg}, \mathrm{Fe}) \mathrm{SiO}_{3}$ Perovskite. In: Perovskite. (Eds. A. Navrotsky and D.J. Weidner). Monograph 45, pp 1-12. Amer. Geophys. Union, Washington DC, 1989.

Yaganeh-Haeri, A, Weidner, D.J. and Ito, E., Single-crystal elastic moduli of magnesium metasilicate perovskite. In: Perovskite. (Eds. A. Navrotsky and D.J. Weidner). Monograph 45, pp 13-25. Amer. Geophys. Union, Washington DC, 1989.

K. Wright and G.D. Price, Dept. of Geological Sciences, University College London, Gower Street, London WC1E 6BT, United Kingdom.

(Received August 25, 1989

Revised October 16, 1989

Accepted October 16, 1989) 